\title{
Hysteretic Optimization
}

\author{
G. Zaránd ${ }^{1,2}$, F. Pázmándi ${ }^{2,3,5}$, K. F. Pál ${ }^{4}$, and G. T. Zimányi ${ }^{3}$ \\ 1 Lyman Physics laboratory, Harvard University, Cambridge MA 02145 \\ ${ }^{2}$ Research Group of the Hungarian Academy of Sciences, Institute of Physics, TU Budapest, H-1521 Hungary \\ ${ }^{3}$ Department of Physics, University of California, Davis, CA 95616 \\ ${ }^{4}$ Institute of Nuclear Research of the Hung. Acad. of Sci., Debrecen, P.O.B 51, H-4001 Hungary \\ ${ }^{5}$ Department of Theoretical Physics, University of Debrecen, P.O. Box 5, H-4010 Hungary
}

(October 29, 2018)

\begin{abstract}
We propose a new optimization method based on a demagnetization procedure well known in magnetism. We show how this procedure can be applied as a general tool to search for optimal solutions in any system where the configuration space is endowed with a suitable 'distance'. We test the new algorithm on frustrated magnetic models and the traveling salesman problem. We find that the new method successfully competes with similar basic algorithms such as simulated annealing.
\end{abstract}

It has been observed long time ago that disordered materials can be brought into a remarkably stable state through annealing, i.e. cooling down the material rather slowly. This simple observation inspired Kirkpatrick, Gelatt, and Vecchi in their pioneering work [i] to investigate how close this annealing procedure takes models with glassy properties to their ground state, and lead them to the invention of the by now widely used simulated annealing (SA) procedure. The SA revealed the crucial role the external noise can play in optimization: Thermal noise can help to escape high-energy local minima. In the present work we investigate another procedure that is commonly used to demagnetize disordered magnets and is experimentally known to result in a very stable state: The application of an oscillating external field (see Figure 1). This procedure makes use of another type of noise which is typical in magnetic systems, namely random external fields. As we shall demonstrate below for various models, a simple generalization of this zero-temperature 'a.c. demagnetization' is able to give systematically better and better approximations to the ground state of these models, and is in many cases 5-10times faster than SA. We shall show how this method can be applied to practically any disordered model, thereby resulting in a new optimization procedure, that we call hysteretic optimization (HO).

Finding optimal solutions of complex problems depending on a large number of parameters is an equally important and difficult task [2]. Examples range from integrated circuit design, through portfolio selection on the stock market [3] and calculating protein folding, to teaching artificial neural networks, to name a few. The simultaneous presence of randomness and frustration is what makes these problems so hard: Disorder is caused by the non-regular dependence of the quality of the solution on the configuration, and frustration is brought in by the competition of mutually exclusive different "good" properties. As a result, on one hand, a naiv search often gets stuck in spurious minima while, on the other hand, comparably good solutions can be found with quite dif- ferent configurations.

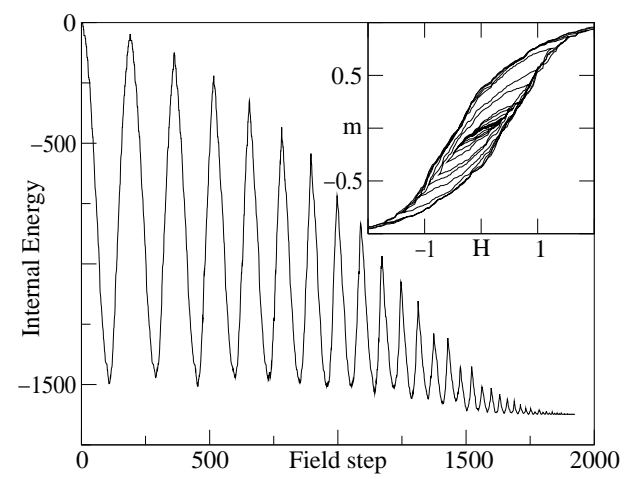

FIG. 1. The total internal energy (without the Zeeman term) of a three dimensional $(L=10)$ Edwards-Anderson spin glass during a.c. demagnetization. The inset shows the corresponding magnetization curve.

It is important to note that most hysteretic systems fulfill the above requirements of complexity. Hysteresis implies the presence of many metastable states caused mainly by disorder. In the case of magnetic materials, the other important ingredient, frustration, is furnished by the magnetostatic interaction, which can be ferro- or antiferromagnetic depending on the relative orientation of the dipoles. This analogy between magnetic sytems and optimization suggests that part of knowledge accumulated through decades by hysteresis research [ [4.5] will eventually prove useful in optimization (and vice versa). Indeed, a simple but very frequently used hys-

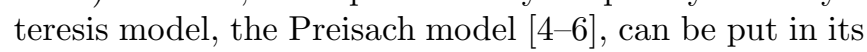
ground state by a.c. demagnetization.

In this letter, we proceed by studying in detail the energetics of a.c. demagnetization for spin glass models. Then we show how this simple method can be modified to obtain a hysteretic optimization technique, which systematically approaches the ground state [7], and we compare the new algorithm with SA. We also present how HO can be combined with cluster renormalization, and apply 
the general formalism of $\mathrm{HO}$ for the traveling salesman problem (TSP).

Many optimization problems can be formulated in terms of interacting Ising spins $(\sigma= \pm 1)$. Therefore we studied first two classical Ising spin glass models [8] with a Hamiltonian:

$$
\mathcal{H}=-\frac{1}{2} \sum_{i, j}^{N} J_{i j} \sigma_{i} \sigma_{j}-H \sum_{i}^{N} \xi_{i} \sigma_{i} .
$$

In the case of the Sherrington-Kirkpatrick (SK) model for any $(i, j)$ pair $J_{i j}=z_{i j} / \sqrt{N}$, where $z_{i j}$ is a random gaussian number with zero mean and unit variance. In the other spin glass we considered, the three dimensional Edwards-Anderson (EA) model, $J_{i j}=z_{i j}$, but only for the nearest-neighbor couplings. The direction $\xi_{i}= \pm 1$ of the external field $H$ randomly changes from site to site. Due to a spin-gauge symmetry, any choice of $\xi_{i}$ is equivalent, as long as it is not correlated with $J_{i j}$. Using this symmetry, and also the analogy of the magnetic energy from equation (11), we call $m=1 / N \sum \xi_{i} \sigma_{i}$ magnetization throughout the paper. These sytems are in the focus of research both in magnetism and in optimization. In magnetism their dynamics and the nature of the ordered state is still highly debated [9, 10]. From the optimization point of view, they are interesting because they are thought to be so-called NP-hard problems [11]. The major difference between the two models lies in the range of interactions. The short-ranged EA model may show some kind of clusterization ("droplets"), which is very unlikely for the infinite-ranged SK model.

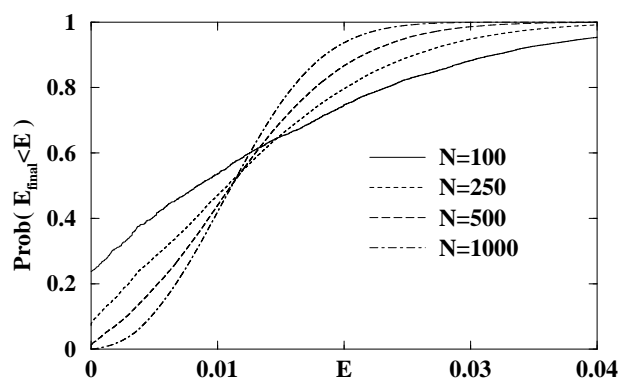

FIG. 2. Size dependence of the integrated energy distribution of states produced by a.c. demagnetization for the SK model $(\gamma=0.9)$. $E$ is the energy per spin measured from the ground state of each sample.

We tested first the energetic properties of a.c. demagnetization on the SK model [12]. Many properties of this model can be calculated exactly [8], and it is an ideal test system. We know, e.g., that its ground state energy is $-0.765 /$ spin as $N \rightarrow \infty$. Starting from a random initial state, a simple 'quench', when randomly chosen unstable spins are aligned one-by-one with their local fields, $h_{i}=\sum_{j} J_{i j} \sigma_{j}+H \xi_{i}$, ends up with (locally) stable states of energy around -0.70 per spin at $H=0$.
We started the a.c. demagnetization with an external field that saturates the spins, i.e. an $H=H_{1}$ large enough that the $\sigma_{i}=\xi_{i}$ state $(m=1)$ be stable. Then we decreased the field $H$ in small steps, each time making a few spins unstable, and 'quenching' the system to a nearby stable state using sequential dynamics. When $H$ reached the 'turning point' $H_{2}=-\gamma_{1} H_{1}$, we started to increase the field again until $H_{3}=-\gamma_{2} H_{2}$, and turned back again and again at points $H_{n}=-\gamma_{n-1} H_{n-1}$, until the amplitude of the loop, $\left|H_{n}\right|$, reached the order of the field steps. The resulting "spiraling" magnetization curve is shown in the inset of Figure 1. We performed a.c. demagnetization with loops which were in average reduced by a factor of $\gamma=\left\langle\gamma_{i}\right\rangle=0.9$ after each turning point (we used $\gamma_{i}$-s between 0.8 and 1.0). We found that, as expected, lower $\gamma$ gives poorer results, but a $\gamma$ even closer to one did not make a considerable improvement. However, randomly varying the turning points, $\gamma_{i}$, increased the efficiency.

Figure 2 shows for different system sizes the probability of finding a configuration close to the ground state 13. of the SK model with a.c. demagnetization. For each $N$ we averaged over several realizations of the couplings and ran a.c. demagnetization many times with different random field directions $\left\{\xi_{i}\right\}$ to generate the distributions presented. The finite intercept at $E=0$ for smaller systems signals a finite probability of finding the ground state, but this probability goes to zero as the size increases: for very large systems a.c. demagnetization typically gives a state with an energy $0.012 \times N$ above the ground state.
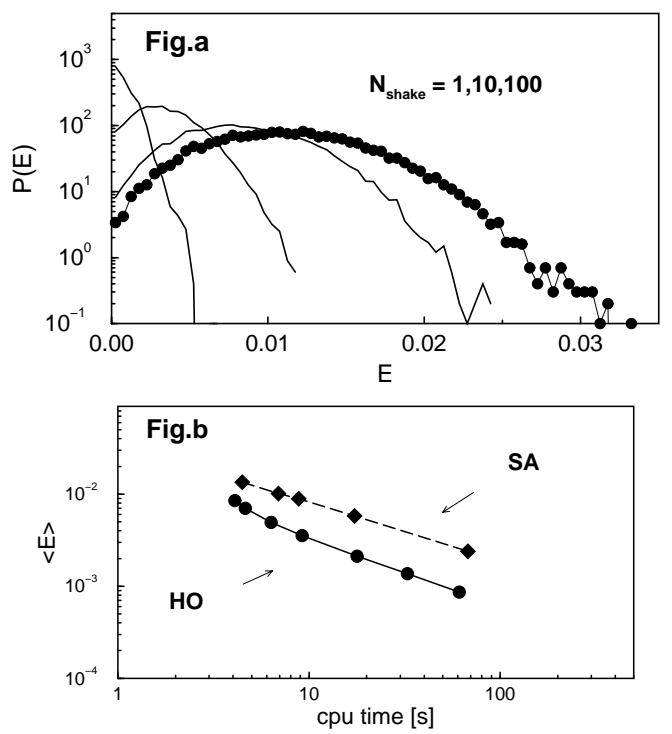

FIG. 3. Fig.a: Probability density of finding a state of an SK model $(N=1000)$ with energy $E$ per spin above the ground state by a.c. demagnetization (symbols) and $\mathrm{HO}$ with 1, 10, and 100 shake ups (solid lines). Fig.b: Average energy per spin measured from the ground state vs. running time for the SK model $(N=1000)$. 
The probability density $P(E)$ of finding a state with energy $E$ produced by a.c. demagnetization for the $N=1000$ SK and the $10 \times 10 \times 10$ EA models is shown by the symbols in Figs. 3. a and 1 . These measurements were performed for a single sample (instance). The a.c. demagnetization gives remarkable improvement with respect to the quench, but in both cases the final state is definitely above the ground state. We emphasize, however, that a.c. demagnetization runs 3-10 times faster than SA (see Figs. 3.b and (1), and can be a viable alternative in applications where a large number of optimizations is required, each of them on relatively smaller systems.

As we mentioned earlier, unlike SA, increasing the factor $\gamma$ closer to one, we only find a limited effect on the quality of solutions obtained by a.c. demagnetization. This is a serious problem, and finding the cause of it is a potentially important subject for future research. We speculate, however, that the answer is hidden in the random, but "frozen" correlations between our noise term $\xi_{i}$ and the generated low-lying states. These correlations may prevent us from reaching certain parts of the phase space. If so, all we have to do is to introduce different $\xi_{i}$-s, since they have different correlations. But we have to do this without destroying the "good" correlations of previous $\xi_{i}$-s.

Maybe the simplest way to achieve this is "shaking up" the system: After finishing an a.c. demagnetization, $H$ being zero, we are free to change $\xi_{i}$ to a different noise "direction" $\xi_{i}^{\prime}$, while the final spin configuration $\sigma_{i}^{0}$ remains stable. Starting from this state, we increase the field in small steps to $H^{m}$, quenching the spins after each step as before. From this point we start a new demagnetization by decreasing the field to $-\gamma_{1} H^{m}$, and turning back e.t.c.. At the and of the shake up we get a new state $\sigma_{i}^{1}$, and $H$ is zero again, so we can choose a new $\xi_{i}^{\prime \prime}$, and start another shake up. While subsequent shake ups with appropriate $H^{m}$ improve the solution on average, it is obviously helpful to get rid of "bad fluctuations", i.e. in case $\sigma_{i}^{1}$ had higher energy than $\sigma_{i}^{0}$, we would start the new shake up from the latter again. It is this combination of a.c. demagnetization and shake ups what we call hysteretic optimization (HO).

The exact choice of the shaking amplitude $H^{m}$ is best obtained by testing it with a few discrete values: too small loops make hardly any change and too large field values take us back to a.c. demagnetization results. Our practice shows that the best choice for $H^{m}$ is around the coercive field, i.e. the field amplitude at which the magnetization reaches zero if started from saturation $(\sim$ 0.3 on Fig. 1).

Figs. 3 and 14 summarize our results of the shake-up HO calculations on the SK and EA models. Apparently, already the first few shake-ups give substantial improvement over a.c. demagnetization. Increasing further the number of shake-ups is less and less effective, but we get systematically closer and closer to the ground state, similar to SA. We would like to emphasize that applying shake-ups after an a.c. demagnetization is straightforward, requires very little extra coding and computer time. A systematic comparison of a.c. demagnetization, shake-up HO, and SA is shown in Figs. 3.b and 4.

In the case of the short-range EA model (inset of Figure (1) shake-ups seem less effective, and for longer running times SA reaches the efficiency of HO. However, typical optimization problems are often closer to the longrange SK model where no particular dimensionality is present (e.g. stock market) [3]. In this case (Fig. 3.b), $\mathrm{HO}$ appears to be better than SA even for long running times.

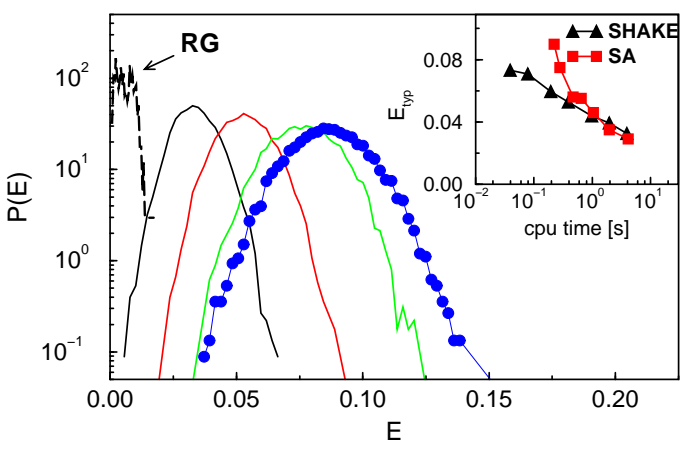

FIG. 4. Probability density of finding a state with energy $E$ per spin above the ground state for the three-dimensional $(L=10)$ Edwards-Anderson model by a.c. demagnetization (symbols) and $\mathrm{HO}$ with 1, 10, and 100 shake ups (solid lines). The dashed line shows results of HO combined with RG. Inset: Typical energies as a function of running times for SA and HO with shake ups.

We have to emphasize that, as well as SA the HO presented in this paper should be considered as a building block, and it can be efficiently combined with other optimization methods, such as genetic algorithms 14, cluster renormalization group (RG) techniques 15, or both [16]. To demonstrate this, we combined HO with the $\mathrm{RG}$ in the following way: We generated clusters from $n(\sim 10-20)$ different low-energy configurations obtained by $\mathrm{HO}$ and using the clusterization technique of Ref. [16]. Using these cluster spins simplifies the problem not just by decreasing the number of degrees of freedom, but also by removing dynamical barriers: turning over a bigger cluster through single spin flips might be energetically expensive because of the strong couplings inside the cluster. For the effective system of cluster spins HO can be applied again to generate $n$ new (cluster) configurations and to define clusters of clusters, and so on. We used this relatively large $n$ [17] on each RG level, and consequently many RG steps, to avoid the necessity of including a genetic algorithm.

It is worth noting that there is a freedom in introducing the external field for the clusters, since the "magnetic 
moment" $\mu$ of a cluster can be arbitrarily chosen. The simplest choice is $\mu=1$ for all clusters, but it might be useful to scale the local moments to insure that small and large clusters can compete with each other. The success of the RG approach for the EA model is due mostly to the short-range nature of the interactions (see Fig. (4). We find that this method is able to reach the ground state in a time comparable to a recent very efficient genetic algorithm developed specifically for this problem [9, 18].

Now we present a general scheme that makes it possible to apply the present algorithm to practically any optimization problem. In an optimization problem one has to minimize some cost function $W(P)$, which is a mapping from some configuration space $\{P\}$ to real numbers. In order to apply $\mathrm{HO}$ to a system we need three important ingredients: $(i)$ Dynamics. This is already sufficient to do SA, however, for the $\mathrm{HO}$ we need also a $(\mathrm{ii}) \mathrm{dis}$ tance $d(P, Q)$ over the configuration space, and (iii) two reference states $R_{ \pm}$. These latter will be used to induce a random external field.

We have a freedom in chosing these ingredients. However, a 'good' dynamics must be such that an elementary step connects configurations with approximately equal cost functions, and its successive applications connect all configurations. Similarly, a 'good' distance corresponding to a given dynamics is such that distances do not change much during a single step.

Having defined all these quantities we define the $\mathrm{HO}$ as the demagnetization of the Hamiltonian

$$
\mathcal{H}(P)=W(P)+\sum_{\alpha= \pm} \alpha H \Theta(\alpha H) d\left(P, R_{\alpha}\right),
$$

whith $\Theta(x)$ being the step function. For the spin problems considered here the configurations are $P=$ $\left\{\sigma_{i}\right\}$, the dynamics is single spin flip, the distance is $d\left(\left\{\sigma_{i}\right\},\left\{\tilde{\sigma}_{i}\right\}\right)=\sum_{i}\left|\sigma_{i}-\tilde{\sigma}_{i}\right|$, and the two reference states are $R_{ \pm}=\left\{ \pm \xi_{1}, \ldots, \pm \xi_{N}\right\}$. With these definitions Eq. (2) reduces to Eq. (11), and the general HO becomes the one we applied to the spin problems. The basic role of the external field in Eq. (2) is to force the system close to the states $R_{ \pm}$as $H \rightarrow \pm \infty$.

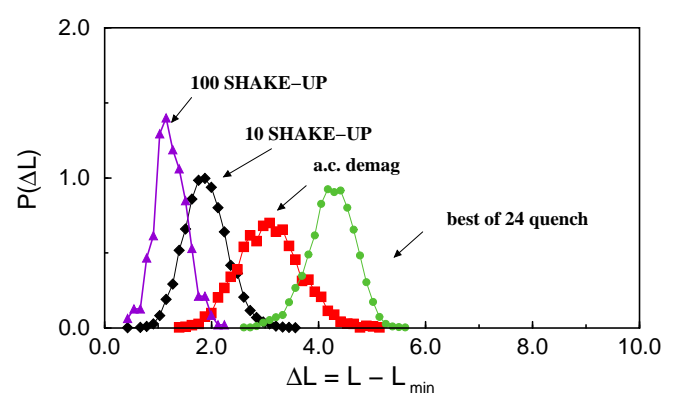

FIG. 5. Distribution of length generated by $\mathrm{HO}$ for a two-dimensional TSP with $N=100$. For comparison, we also show the best of 24 quenches, taking the same time as a.c. demagnetization.
Now we demonstarate how this method can be applied for the TSP. In this classical optimization problem the aim is to find the shortest path that goes through each of a given set of $i=1, . ., N$ cities, visiting just once every one of them. In this problem the randomness is in the location $\mathbf{x}_{i}$ of the cities. The naive strategy of going always to the closest unvisited city fails, because typically there is a huge penalty for the last trip home - giving the major source of frustration. The configuration space is just given by all possible permutations $P$ of the cities, and the cost function is simply the total length of the path, $W(P)=\sum_{i=1}^{N}\left|\mathbf{x}_{P(i)}-\mathbf{x}_{P(i+1)}\right|$. As an elementary step we used the interchange of two cities [19], and a distance

$$
d(P, Q) \equiv \sum_{i}^{N}\left|\boldsymbol{\Delta}_{i}(P)-\boldsymbol{\Delta}_{i}(Q)\right|
$$

where $\boldsymbol{\Delta}_{i}(P)=\mathbf{x}_{P(i+1)}-\mathbf{x}_{P(i)}$ denotes the vector connecting the cities $P(i)$ and $P(i+1)$. Our results for the traveling salesman are summarized in Fig. 5. We find a systematic improvement as the number of shake ups increases, and get better and better results in this case too.

There is one more aspect of $\mathrm{HO}$ we would like to emphasize. SA has the nice property of self-consistently telling us how the annealing schedule should be set: the specific heat is proportional to the fluctuations, so one might want to spend more time in the temperature regions of high specific heat. A similar measure of accuracy for $\mathrm{HO}$ might be the measured Preisach function of the system. This function can be obtained numerically from first order reversal curves [5], and once at hand, it can guide the annealing of the loops. We believe that this function has a lot of information about the metastable states of the system, although admittedly future research is needed to identify these features.

Acknowledgements: This research has been supported by NSF Grants No. DMR99-81283, DMR97-14725 and DMR99-85978 and Hungarian Grants No. OTKA F030041, T029813, and 29236.

[1] S. Kirkpatrick, C.D. Gelatt, Jr., and M.P. Vecchi, Science 220, 671 (1983).

[2] C.H. Papadimitriou and Steiglitz, Combinatorial Optimization: Algorithms and Complexity (Prentice Hall, 1982).

[3] S. Gallucio, J.-P. Bouchaud, and M. Potters, Physica 259A, 449 (1998), A. Gábor and I. Kondor, Physica 274A, 222 (1999)

[4] G. Bertotti, Hysteresis and Magnetism (Academic Press, 1998).

[5] E. Della Torre, Magnetic Hysteresis (IEEE Press, 1999).

[6] F. Preisach, Z. Phys., 94, 277 (1935).

[7] We have no proof of convergence to the ground sate.

[8] M. Mézard, G. Parisi, and M.A. Virasoro, Spin-Glass Theory and Beyond (World Scientific, 1987). 
[9] M. Palassini and A.P. Young, Phys. Rev. Lett. 85, 3017 (2000); J.-F. Bouchaud (unpublished).

[10] A. A. Middleton, Phys. Rev. B 63, 060202(R) (2001).

[11] A. K. Hartmann, Physica A 224, 480 (1996)

[12] G. Bertotti and M. Pasquale, J. Appl. Phys. 67, 5255 (1990).

[13] We used the method of [18 to obtain the ground state for all models.

[14] D.E. Goldberg, Genetic Algorithms in Search, Optimization and Machine Learning (Addison-Wesley, Reading, 1989).

[15] N. Kawashima and M. Suzuki, J. Phys. A 25, 1055(1992)

[16] J. Houdayer and O.C. Martin, Phys. Rev. Lett. 83, 1030 (1999), used RG combined with a genetic algorithm.

[17] This cluster RG can be solved exactly for the onedimensional EA spin glass.

[18] K. F. Pal, Physica (Amsterdam) 223A, 283 (1996); ibid. 233A, 60 (1996).

[19] While more complicated dynamical steps [1] can be more efficient, here we chose a less efficient dynamics which allows one to more clearly judge the performance of HO. The ground state of the TSP was found using twist dynamics 值. 